\title{
BMJ Open Vasopressor use following traumatic injury: protocol for a systematic review
}

\begin{abstract}
Mathieu Hylands, ${ }^{1}$ Augustin Toma, ${ }^{2}$ Nicolas Beaudoin, ${ }^{3}$ Anne-Julie Frenette, ${ }^{4}$ Frederick D'Aragon, ${ }^{3,5}$ Emilie Belley-Côté, ${ }^{2,6}$ Morten Hylander, ${ }^{7}$ François Lauzier, ${ }^{8}$ Reed Alexander Siemieniuk, ${ }^{2}$ Emmanuel Charbonney, ${ }^{4}$ Joey Kwong, ${ }^{9}$ Jon Henrik Laake, ${ }^{10}$ Gordon Guyatt, ${ }^{2}$ Per Olav Vandvik, ${ }^{11}$ Bram Rochwerg, ${ }^{2}$ Robert Green, ${ }^{12}$ Ian Ball, ${ }^{13}$ Damon Scales, ${ }^{14}$ Srinivas Murthy, ${ }^{15}$ Sandro Rizoli, ${ }^{16}$ Pierre Asfar, ${ }^{17}$ François Lamontagne ${ }^{5,6}$
\end{abstract}

To cite: Hylands M, Toma A, Beaudoin N, et al. Vasopressor use following traumatic injury: protocol for a systematic review. BMJ Open 2017;7:e014166. doi:10.1136/bmjopen-2016014166

- Prepublication history and additional material is available. To view please visit the journal (http://dx.doi.org/ 10.1136/bmjopen-2016014166).

Received 23 November 2016 Revised 3 January 2017 Accepted 1 February 2017

CrossMark

For numbered affiliations see end of article.

Correspondence to Dr François Lamontagne; francois.lamontagne@ usherbrooke.ca

\section{ABSTRACT}

Introduction: Worldwide, traumatic casualties are projected to exceed 8 million by year 2020 .

Haemorrhagic shock and brain injury are the leading causes of death following trauma. While intravenous fluids have traditionally been used to support organ perfusion in the setting of haemorrhage, recent investigations have suggested that restricting fluid therapy by tolerating more severe hypotension may improve survival. However, the safety of permissive hypotension remains uncertain, particularly among patients who have suffered a traumatic brain injury. Vasopressors preferentially vasoconstrict blood vessels that supply nonvital organs and capacitance vessels, thereby mobilising the unstressed blood volume. Used as fluid-sparing adjuncts, these drugs can complement resuscitative measures by correcting hypotension without diluting clotting factors or increasing the risk for tissue oedema.

Methods and analysis: We will identify randomised control trials comparing early resuscitation with vasopressors versus placebo or standard care in adults following traumatic injury. Data sources will include MEDLINE, EMBASE, CENTRAL, clinical trial registries and conference proceedings. Two reviewers will independently determine trial eligibility. For each included trial, we will conduct duplicate independent data extraction and risk of bias assessment. We will assess the overall quality of the data for each individual outcome using the GRADE approach.

Ethics and dissemination: We will report this review in accordance with the PRISMA statement. We will disseminate our findings at critical care and trauma conferences and through a publication in a peerreviewed journal. We will also use this systematic review to create clinical guidelines (http://www.magicapp.org), which will be disseminated in a standalone publication. Trial registration number: CRD42016033437.

\section{INTRODUCTION}

\section{Description of the problem}

Traumatic injuries caused over 5 million deaths worldwide in $2010^{1}$ and casualities are

\section{Strengths and limitations of this study}

- This will be the first systematic review of vasopressors in early trauma resuscitation to incorporate a detailed search strategy of published and unpublished studies, explicit inclusion and exclusion criteria, duplicate independent screening, data extraction, and risk of bias assessment.

- Overall quality of available evidence will be assessed using the Grading of Recommendations, Assessment, Development and Evaluation (GRADE) approach.

- Conclusions will be limited by the number and quality of available studies.

projected to exceed 8 million by $2020 .^{2}$ Haemorrhagic shock is the leading cause of preventable death following trauma. ${ }^{3}$ Fluids have traditionally been used to support organ perfusion in the setting of haemorrhage, but recent investigations have highlighted the risks of excessive fluid administration. In a landmark trial, hypotensive patients with penetrating torso trauma were more likely to be discharged alive from hospital when fluid resuscitation was withheld until arrival to the operating theatre. ${ }^{4}$ These results are concordant with data from a subsequent 90-patient trial on fluid use during trauma surgery, where a mean arterial pressure target of 50 vs $65 \mathrm{~mm} \mathrm{Hg}$ significantly decreased blood product use without increasing 30-day mortality. ${ }^{5}$ Recent trauma guidelines have incorporated restrictive fluid strategies, referred to as permissive hypotension, into their recommendations. ${ }^{67}$ However, as pointed out in two systematic reviews, ${ }^{8}{ }^{9}$ the safety of permissive hypotension remains uncertain. This may be particularly true among patients with concomitant traumatic brain injury in whom hypotension is associated with increased mortality. ${ }^{4}$ 10-12 


\section{Description of the intervention}

Vasopressors are vasoactive agents that induce vasoconstriction and increase arterial pressure. ${ }^{13}$ They also vasoconstrict capacitance vessels, thereby mobilising the unstressed blood volume and increasing venous return. ${ }^{14}$ Vasopressors can rapidly correct hypotension in an effort to maintain end-organ perfusion in the face of hypovolaemic shock. ${ }^{15}$ Used as fluid-sparing adjuncts to resuscitation, vasopressors can complement resuscitative measures by correcting hypotension without diluting clotting factors or increasing the risk for tissue oedema. $^{16}$

In spite of this potential role as fluid-sparing adjuncts, ${ }^{16}$ vasopressors potentiate vasoconstriction and may therefore worsen hypoperfusion, despite high blood pressure values. ${ }^{17}$ Thus, trauma guidelines restrict vasopressor use to cases of severe hypotension refractory to fluid therapy, ${ }^{6}$ but wide practice variations exist. ${ }^{18}$ In the absence of high-quality evidence, experts recommend limiting vasopressors to patients unresponsive to fluid therapy, ${ }^{7}$ while recognising that a significant knowledge gap exists. ${ }^{6}$

\section{Why is it important to conduct this review?}

Given the well-recognised risks of excessive fluid administration, ${ }^{4}{ }^{19-22}$ healthcare providers are left with few alternatives for unstable trauma patients in whom hypotension may be deleterious. Vasopressors are increasingly perceived as a complementary strategy, ${ }^{18}$ but their associated harms and benefits have never been conclusively examined. No systematic review has yet focused on the efficacy or safety of vasopressors in trauma.

\section{Research question}

Does the early use of vasopressors improve the survival of victims of traumatic injury, compared with standard care which incorporates vasopressors only once other modalities of support have failed?

\section{METHODS AND ANALYSIS}

\section{Criteria for selecting studies for this review}

\section{Types of studies}

We will include all randomised controlled trials and controlled observational studies (case-control or cohort). We will exclude case reports and case series. We will not use restrictions based on language, methodological quality, publication status or year of publication.

\section{Types of participants}

Our population of interest consists of adult victims of acute, non-iatrogenic traumatic injury (blunt or penetrating). We will include studies reporting diverse populations, such as severe burns, if there are extractable data on the trauma subgroup or if the non-trauma subgroup constitutes $<10 \%$ of the total study population. We will exclude paediatric ( $<16$ years) and animal studies.

\section{Types of interventions}

The interventions of interest are the administration of any vasopressor (epinephrine, norepinephrine, dopamine, phenylephrine, ephedrine vasopressin or vasopressin analogues) during early trauma resuscitation. We will include studies that consider cardiac inotropes (eg, milrinone) if these account for $<10 \%$ of the vasopressor group. We will exclude studies that report vasopressor use exclusively during the postoperative phase, after arrival in the intensive care unit or $>24$ hours after arrival at the trauma bay. We will also exclude studies that rely on cointerventions not available in the early phases of care, such as cerebral perfusion pressure monitoring.

\section{Types of outcome measures}

We will exclude studies where follow-up was $<24$ hours. The primary outcome will be short-term mortality at longest follow-up up to 90 days. Other outcomes will be long-term mortality beyond 90 days, fluid and blood product requirements during the early resuscitation period, requirements for acute (up to 90 days) or chronic (beyond 90 days) renal replacement therapy, duration of renal replacement therapy, duration of mechanical ventilation, incidence of acute kidney injury, incidence of vasopressor-associated adverse events (newonset cardiac arrhythmia, digit, limb or skin ischaemia, mesenteric ischaemia and myocardial ischaemia), neurological outcome and long-term quality of life.

\section{Search methods for the identification of studies}

We will perform a search of the following databases for relevant studies: MEDLINE, EMBASE and the Cochrane Central Register of Controlled Trials (CENTRAL). The search strategy specific to MEDLINE is included as online supplementary appendix 1 . We will perform similar searches, with keywords adapted to specific database dictionaries.

\section{Additional search methods (grey literature)}

We will screen reference lists of included studies and relevant reviews for eligible articles. We will also manually screen conference proceedings from 2005 for the following scientific meetings: Society of Critical Care Medicine, European Society of Intensive Care Medicine, International Society of Intensive Care and Emergency Medicine, American Thoracic Society, American Association for the Surgery of Trauma, Eastern Association for the Surgery of Trauma, European Society for Trauma and Emergency Surgery, Shock Society, European Shock Society and the American College of Chest Physicians. We will also search ClinicalTrials.gov for any relevant ongoing or unpublished trials. Whenever possible, we will contact authors to obtain additional data.

\section{Study records}

Pairs of reviewers will independently screen titles and abstracts using a pretested electronic screening form 
(Covidence web platform: http://www.COVIDENCE. org). We will include articles for full-text review unless both reviewers deem them irrelevant. Pairs of reviewers will then independently screen all full-text articles using specific eligibility criteria via pretested electronic screening tools (Covidence). We will resolve disagreements by consensus or third-reviewer adjudication if necessary. We will report chance-corrected agreement using Cohen's $\kappa$ for full-text eligibility screening.

\section{Data collection}

Teams of two reviewers will perform data extraction independently and in duplicate using pretested data collection forms (Covidence). We will collect information pertaining to study design, patient baseline characteristics, intervention and comparator, clinical outcomes and risk of bias. Conflicts will be resolved by consensus or third-abstractor adjudication if necessary.

\section{Risk of bias assessment}

For randomised controlled trials, we used a modified version of the Cochrane Collaboration tool to assess the risk of bias of individual studies. ${ }^{23}$ This tool addresses the following domains: randomisation, allocation concealment, blinding of patients, healthcare providers, data collectors, outcome assessors and data analysts, loss to follow-up, selective outcome reporting and other risks of bias.

For observational studies, we will use the risk of bias tools developed by the 'Clinical Advances through Research and Information Technology' (CLARITY) group at McMaster university (https://distillercer.com/ resources/). ${ }^{24}{ }^{25}$ These tools evaluate the selection of the intervention and control groups, the adequacy of assessment of prognostic factors, exposure and clinical outcomes, statistical adjustment and/or matching, follow-up, similarity of cointerventions between groups and other risks of bias.

Studies with one or more domain assessed as a potential source of bias will be considered overall at high risk of bias. We will assess the overall quality of the data for each individual outcome using the GRADE approach. ${ }^{26}$

\section{Summarising data and treatment effect}

We will include the results of clinically homogeneous studies in a meta-analysis using the Review Manager software (Review Manager 5.3). We will use a random-effects model $^{27}$ and the inverse variance method to calculate individual study weights. Randomised controlled trials and observational studies will be meta-analysed separately and presented as forest plots. For dichotomous outcomes, summary effect measures will be pooled from individual study ORs and presented as risk ratios with $95 \%$ CIs. For continuous outcomes, summary effect measures will be presented as mean differences with 95\% CIs. Health-related quality-of-life outcomes may be reported using a variety of different scales. In this situation, we will use the methods of reporting suggested by
Thorlund et al. $^{28}$ Their recommendations encourage the reporting of summary measures as a number needed to treat using at least two complementary methods, in order to improve the interpretability of results.

We will provide qualitative summaries of outcomes for which quantitative summaries are deemed inappropriate and justify our rationale. We will use trial sequential analysis to assess the risk of random errors. ${ }^{29}$

\section{Assessment of heterogeneity}

We will assess and report heterogeneity quantitatively the $\mathrm{I}^{2}$ statistic and perform a $\chi^{2}$ test for homogeneity. Irrespective of the degree of heterogeneity, we will perform prespecified exploratory subgroup analyses.

\section{Subgroup analysis and investigation of heterogeneity}

We will perform the following comparisons to identify potential subgroup effects. (1) Patients with severe traumatic brain injury (Glasgow Coma Scale $\leq 8$ ) versus those without (Glasgow Coma Scale $>8$ ), hypothesising that patients with severe traumatic brain injury benefit more from vasopressors, given their vulnerability to hypotension and the risk of harm associated with fluid resuscitation and permissive hypotension. (2) Patients with blunt trauma versus those with penetrating trauma, hypothesising that there may be a greater benefit for blunt trauma victims, as they may be less likely to benefit from transient hypotension to control a discrete source of bleeding as in penetrating trauma. (3) Patients aged $\leq 45$ years versus those aged $>45$ years (if within study subgroup data available) or studies where the median age is $>45$ versus $\leq 45$ years hypothesising that older patients may be more vulnerable to hypotension and therefore receive supplemental benefit from vasopressor therapy. (4) Academic trauma centre versus community setting, hypothesising that vasopressors may be more beneficial in community settings where transport times and in-hospital delays likely expose patients to resuscitative measures for prolonged periods of time, thereby increasing the risks associated with transient hypotension. (5) High or unclear risk of bias versus low risk of bias, hypothesising that studies with high or unclear risk of bias may overestimate the benefits of early vasopressor therapy. (6) Early studies versus more recent studies (within the last 10 years) hypothesising that recent studies report greater benefit of early vasopressor use.

For between-study comparisons, we will require at least five studies, with each subgroup represented by at least two studies, to undertake a subgroup analysis. A minimum of two studies will be required to conduct a within-study subgroup analysis.

\section{Assessment of reporting bias}

If we include 10 or more studies in a meta-analysis, we will assess the potential for publication bias visually using a funnel plot and statistically using Egger's test ${ }^{30}$ for continuous outcomes and the arcsine test ${ }^{31}$ for dichotomous outcomes. 


\section{Assessment of confidence in estimates of effect}

We will use the Grading of Recommendations, Assessment, Development and Evaluation (GRADE) framework $^{32}$ to report our overall confidence in estimates of effect. This method considers the overall risk of bias, ${ }^{33}$ imprecision, ${ }^{34}$ inconsistency, ${ }^{35}$ indirectness ${ }^{36}$ and likelihood of publication bias ${ }^{37}$ to judge the overall quality of evidence for each outcome. Quality of evidence is rated 'very low', 'low', 'moderate' or 'high'. Randomised controlled trials provide high-quality evidence, while observational studies yield low-quality evidence. Trials can be rated down according to the above-mentioned criteria. Observational studies can be rated up in the presence of a large magnitude of the association, a dose-response gradient or if all unaccounted confounders increase confidence in estimates of effect.

The findings of this review will be summarised and presented with a summary of findings table with an explicit judgement of quality of evidence for each outcome across studies. ${ }^{38}$

\section{DISCUSSION}

Traumatic injuries remain one of the leading causes of mortality worldwide. For many patients, healthcare providers are left with few safe options for haemodynamic support. Although restrictive fluid strategies may be beneficial for victims of penetrating torso injuries, ${ }^{4}$ this strategy is unsafe for patients with signs of neurological injury. Vasopressors could provide a much-needed complementary means of haemodynamic support in this vulnerable population. Given the theoretical risks associated with these agents, a rigorous evaluation of the published evidence is required to guide clinical practice.

This methodologically rigorous systematic review will summarise the existing evidence on the efficacy and safety of early vasopressor use following traumatic injury. Strengths of this review include duplicate risk of bias assessment, evaluation of the quality of evidence using the GRADE approach, a detailed search of published studies and grey literature, predefined study eligibility criteria and a priori subgroup hypotheses.

\section{DISSEMINATION}

We will report this review in accordance with the PRISMA statement. ${ }^{39}$ This protocol is reported in accordance with the Preferred Reporting Items for Systematic Reviews and Meta-Analyses Protocols (PRISMA-P) guidelines. ${ }^{40}$ We will disseminate our findings by emitting clinical guidelines using the MagiApp (http://www.magicapp.org) as well as conference presentations and publication in a peer-reviewed journal.

\section{Author affiliations}

${ }^{1}$ Department of Surgery, Université de Sherbrooke, Sherbrooke, Quebec, Canada

${ }^{2}$ McMaster University, Clinical Epidemiology and Biostatistics, Hamilton, Ontario, Canada
${ }^{3}$ Department of Anaesthesiology, Université de Sherbrooke, Sherbrooke, Quebec, Canada

${ }^{4}$ Hôpital du Sacré-Coeur de Montréal, Université de Montréal, Montréal,

Quebec, Canada

${ }^{5}$ Centre de recherche du CHU de Sherbrooke, Sherbrooke, Quebec, Canada

${ }^{6}$ Department of Medicine, Université de Sherbrooke, Sherbrooke, Quebec,

Canada

${ }^{7}$ Copenhagen University Hospital Rigshospitalet, Copenhagen, Denmark

${ }^{8}$ Population Health and Optimal Health Practives Research Unit (Trauma-

Emergency-Critical Care Medicine), Centre de Recherche du CHU de Québec

- Université Laval, Quebec, Quebec, Canada

${ }^{9}$ Wuhan University, Center for Evidence-Based and Translational Medicine Zhongnan Hospital, Wuhan, China

${ }^{10}$ Oslo Universitetssykehus Ulleval, Oslo, Norway

${ }^{11}$ Norwegian Knowledge Centre for the Health Services, Oslo, Norway

${ }^{12}$ Department of Critical care, Dalhousie University, Halifax, Nova Scotia,

Canada

${ }^{13}$ London Health Sciences Centre, London, Ontario, Canada

${ }^{14}$ Department of Critical Care, University of Toronto, Toronto, Ontario, Canada

${ }^{15}$ Department of Paediatrics, University of British Columbia, Vancouver, British Columbia, Canada

${ }^{16}$ Division of General Surgery, St. Michael's Hospital, University of Toronto,

Toronto, Ontario, Canada

${ }^{17}$ Centre Hospitalier Universitaire d’Angers, Angers, France

Twitter Follow lan Ball @Ball

Acknowledgements The authors are indebted to Jean Maragno and Lois Cottrell for their guidance in designing and carrying out our search strategy.

Collaborators Canadian Critical Care Trials Group (CCCTG).

Contributors FLam and $\mathrm{MH}$ conceived the idea for this systematic review. FLam, MH, AT, NB, A-JF, FDA, EB-C, MH, FLau, RAS, EC, JHL, GG, POV, BR, DS, SM, SR and PA developed the methodology for the systematic review protocol. The manuscript was drafted by $\mathrm{MH}$ and FLam and revised by $A T$ NB, A-JF, FDA, EB-C, MHM, FLau, RAS, EC, JK, JHL, GG, POV, BR, RG, IB, $\mathrm{DS}, \mathrm{SM}, \mathrm{SR}$ and PA. MH developed the search strategy and will screen potential studies, perform duplicate independent data extraction, risk of bias assessment, GRADE assessment with AT, NB, A-JF and JK. MH will conduct the data synthesis. FLam will act as a third reviewer and arbitrator if necessary. FLam is the guarantor of the review.

Competing interests None declared.

Ethics approval Not required.

Provenance and peer review Not commissioned; externally peer reviewed.

Data sharing statement The data and analyses of the systematic review will be available to the public

Open Access This is an Open Access article distributed in accordance with the Creative Commons Attribution Non Commercial (CC BY-NC 4.0) license, which permits others to distribute, remix, adapt, build upon this work noncommercially, and license their derivative works on different terms, provided the original work is properly cited and the use is non-commercial. See: http:// creativecommons.org/licenses/by-nc/4.0/

\section{REFERENCES}

1. Lozano R, Naghavi M, Foreman K, et al. Global and regional mortality from 235 causes of death for 20 age groups in 1990 and 2010: a systematic analysis for the Global Burden of Disease Study 2010. Lancet 2012;380:2095-128.

2. Murray CJ, Lopez AD. Alternative projections of mortality and disability by cause 1990-2020: Global Burden of Disease Study Lancet 1997;349:1498-504.

3. Tien HC, Spencer F, Tremblay LN, et al. Preventable deaths from hemorrhage at a level I Canadian trauma center. J Trauma Acute Care Surg 2007;62:142-6.

4. Bickell WH, Wall MJ Jr, Pepe PE, et al. Immediate versus delayed fluid resuscitation for hypotensive patients with penetrating torso injuries. N Engl J Med 1994;331:1105-9. 
5. Morrison CA, Carrick MM, Norman MA, et al. Hypotensive resuscitation strategy reduces transfusion requirements and severe postoperative coagulopathy in trauma patients with hemorrhagic shock: preliminary results of a randomized controlled trial. J Trauma 2011;70:652-63.

6. Spahn DR, Bouillon B, Cerny V, et al. Management of bleeding and coagulopathy following major trauma: an updated European guideline. Crit Care 2013;17:R76.

7. Rotondo MF, Fildes J, Brasel K, et al. ATLS Advanced Trauma Life Support for Doctors-Student Course Manual. 9th edn. American College of Surgeons, 2012

8. Kwan I, Bunn F, Chinnock P, et al. Timing and volume of fluid administration for patients with bleeding. Cochrane Database Syst Rev 2014;3:CD002245.

9. Wang $\mathrm{CH}, \mathrm{Hsieh} \mathrm{WH}, \mathrm{Chou} \mathrm{HC}$, et al. Liberal versus restricted fluid resuscitation strategies in trauma patients: a systematic review and meta-analysis of randomized controlled trials and observational studies. Crit Care Med 2014;42:954-61.

10. Dutton RP, Mackenzie CF, Scalea TM. Hypotensive resuscitation during active hemorrhage: impact on in-hospital mortality. J Trauma 2002;52:1141-6.

11. McHugh GS, Engel DC, Butcher I, et al. Prognostic value of secondary insults in traumatic brain injury: results from the IMPACT Study. J Neurotrauma 2007;24:287-93.

12. Chesnut RM, Marshall LF, Klauber MR, et al. The role of secondary brain injury in determining outcome from severe head injury. J Trauma 1993;34:216-22.

13. Hollenberg SM. Vasoactive drugs in circulatory shock. Am J Respir Crit Care Med 2011:183:847-55.

14. Rivers E, Nguyen B, Havstad S, et al. Early goal-directed therapy in the treatment of severe sepsis and septic shock. N Engl J Med 2001;345:1368-77.

15. Poloujadoff M-P, Borron SW, Amathieu R, et al. Improved survival after resuscitation with norepinephrine in a murine model of uncontrolled hemorrhagic shock. Anesthesiology 2007;107:591-6.

16. Beloncle $\mathrm{F}$, Meziani $\mathrm{F}$, Lerolle $\mathrm{N}$, et al. Does vasopressor therapy have an indication in hemorrhagic shock? Ann Intensive Care 2013;3:13

17. Bellomo R, Wan L, May C. Vasoactive drugs and acute kidney injury. Crit Care Med 2008;36:S179-86.

18. Fangio $P$, Asehnoune $K$, Edouard $A$, et al. Early embolization and vasopressor administration for management of life-threatening hemorrhage from pelvic fracture. $J$ Trauma Acute Care Surg 2005;58:978-84.

19. Haut ER, Kalish BT, Cotton BA, et al. Prehospital intravenous fluid administration is associated with higher mortality in trauma patients: a National Trauma Data Bank analysis. Ann Surg 2011;253:371-7.

20. Madigan MC, Kemp CD, Johnson JC, et al. Secondary abdominal compartment syndrome after severe extremity injury: are early, aggressive fluid resuscitation strategies to blame? J Trauma 2008;64:280-5.

21. Maegele M, Lefering R, Yucel N, et al. Early coagulopathy in multiple injury: an analysis from the German Trauma Registry on 8724 patients. Injury 2007;38:298-304.

22. Schnuriger $\mathrm{B}$, Inaba $\mathrm{K}, \mathrm{Wu} \mathrm{T}$, et al. Crystalloids after primary colon resection and anastomosis at initial trauma laparotomy: excessive volumes are associated with anastomotic leakage. $J$ Trauma 2011;70:603-10.

23. Higgins JPT, Green S. Cochrane handbook for systematic reviews of interventions. Version 5.1. 0 [updated March 2011]. The Cochrane Collaboration, 2011. http://www cochrane-handbook org 2014

24. Tool to Assess Risk of Bias in Case-control Studies. https:// distillercer.com/wp-content/uploads/2014/02/Tool-to-Assess-Riskof-Bias-in-Case-Control-Studies-Aug-21_2011.doc

25. Tool to Assess Risk of Bias in Cohort Studies. https://distillercer. com/wp-content/uploads/2014/02/Tool-to-Assess-Risk-ofBias-in-Cohort-Studies.doc

26. Guyatt GH, Oxman AD, Vist GE, et al. GRADE: an emerging consensus on rating quality of evidence and strength of recommendations. BMJ 2008;336:924-6.

27. DerSimonian R, Laird N. Meta-analysis in clinical trials. Control Clin Trials 1986;7:177-88.

28. Thorlund K, Walter SD, Johnston BC, et al. Pooling health-related quality of life outcomes in meta-analysis-a tutorial and review of methods for enhancing interpretability. Res Synth Methods 2011;2:188-203.

29. Brok J, Thorlund K, Gluud C, et al. Trial sequential analysis reveals insufficient information size and potentially false positive results in many meta-analyses. J Clin Epidemiol 2008;61:763-9.

30. Egger M, Smith GD, Schneider M, et al. Bias in meta-analysis detected by a simple, graphical test. BMJ 1997;315:629-34.

31. Rücker G, Schwarzer G, Carpenter J. Arcsine test for publication bias in meta-analyses with binary outcomes. Stat Med 2008;27:746-63.

32. Balshem $\mathrm{H}$, Helfand $\mathrm{M}$, Schünemann $\mathrm{HJ}$, et al. GRADE guidelines: 3. Rating the quality of evidence. J Clin Epidemio 2011;64:401-6

33. Guyatt GH, Oxman AD, Vist G, et al. GRADE guidelines: 4. Rating the quality of evidence-study limitations (risk of bias). $J$ Clin Epidemiol 2011;64:407-15.

34. Guyatt GH, Oxman AD, Kunz R, et al. GRADE guidelines: 6. Rating the quality of evidence-imprecision. J Clin Epidemiol 2011;64:1283-93

35. Guyatt GH, Oxman AD, Kunz R, et al. GRADE guidelines: 7. Rating the quality of evidence-inconsistency. J Clin Epidemiol 2011;64:1294-302.

36. Guyatt GH, Oxman AD, Kunz R, et al. GRADE guidelines: 8. Rating the quality of evidence-indirectness. J Clin Epidemiol 2011;64:1303-10.

37. Guyatt GH, Oxman AD, Montori V, et al. GRADE guidelines. 5. Rating the quality of evidence-publication bias. $J$ Clin Epidemiol 2011;64:1277-82.

38. Guyatt G, Oxman AD, Akl EA, et al. GRADE guidelines: 1. Introduction-GRADE evidence profiles and summary of findings tables. J Clin Epidemiol 2011:64:383-94.

39. Moher D, Liberati A, Tetzlaff J, et al. Preferred reporting items for systematic reviews and meta-analyses: the PRISMA statement. Ann Intern Med 2009;151:264-9.

40. Moher D, Shamseer L, Clarke M, et al. Preferred reporting items for systematic review and meta-analysis protocols (PRISMA-P) 2015 statement. Syst Rev 2015;4:1. 